\title{
BMJ Open Chemoattractant receptor-homologous molecule expressed on Th2 cells (CRTH2) antagonists in asthma: a systematic review and meta- analysis protocol
}

Wei Liu, Jie Min, Hongli Jiang, Bing Mao

To cite: Liu W, Min J, Jiang H, et al. Chemoattractant receptor-homologous molecule expressed on Th2 cells (CRTH2) antagonists in asthma: a systematic review and metaanalysis protocol. BMJ Open 2018;8:e020882. doi:10.1136/ bmjopen-2017-020882

- Prepublication history and additional material for this paper are available online. To view these files, please visit the journal online (http://dx.doi org/10.1136/bmjopen-2017020882).

Received 30 November 2017 Revised 12 March 2018 Accepted 15 March 2018

\section{Check for updates}

Division of Respiratory Medicine, Department of Integrated Traditional Chinese and Western Medicine, West China Hospital, Sichuan University, Chengdu, China

Correspondence to Dr Hongli Jiang; doc_jh|@163.com

\section{ABSTRACT}

Introduction More than 20 orally bioavailable chemoattractant receptor-homologous molecule expressed on Th2 cells (CRTH2) antagonists have moved forward to clinical development in recent years for the treatment of asthma. However, evidence from individual randomised controlled trials (RCTs) has demonstrated inconsistent results in their efficacy and safety. Methods and analysis PubMed/Medline, Embase, Web of Science, Cochrane Database of Systematic Reviews, Global Index Medicus, Cochrane Central Register of Controlled Trials and Scopus will be searched from inception to 30 December 2017 for eligible RCTs, with additional studies being identified by manual searches. The study eligibility, data extraction and quality appraisal will be performed by two independent reviewers. Studies deemed fit for inclusion will be assessed using Cochrane Collaboration risk of bias tool. To generate more accurate analyses, Grading of Recommendations Assessment, Development and Evaluation will be used to grade the evidence. We will use the $\chi^{2}$ test and the $\mathrm{I}^{2}$ statistic to assess heterogeneity. The metaregression and subgroup analyses will be undertaken in the presence of heterogeneity. The potential for publication bias will be examined using funnel plots.

Ethics and dissemination The current study is based on published data, thus ethical approval is not a requirement. The results of this study will be reported in an open-access peer-reviewed publication or will be disseminated as conference proceedings. This systematic review will increase the understanding of the application of CRTH2 antagonists in patients with asthma, which may help to establish and identify specific gaps in the evidence informing a future agenda for asthma research, policy and practice.

Trial registration number CRD42017079342.

\section{INTRODUCTION}

Asthma is a chronic inflammatory lung disease affecting 235-330million people worldwide. It represents a major societal health problem. ${ }^{1}$ The goals of current guidelines for asthma management are to achieve
Strengths and limitations of this study

- To the best of our knowledge, this is the first systematic review and meta-analysis comprehensively summarising the available evidence on the effectiveness and safety of chemoattractant receptor-homologous molecule expressed on Th2 cells (CRTH2) antagonists in patients with asthma.

- Subgroup analyses will comprehensively address the influence of patient characteristics (inflammation phenotype, disease severity, allergic/atopic status) and interventions (pharmacological mechanism) on the efficacy of CRTH2 antagonists in asthma treatment, where sufficient data are available.

- As there are no head-to-head trials of CRTH2 antagonists, the current meta-analysis cannot directly assess the efficacy of $\mathrm{CRTH} 2$ antagonists relative to each other.

- By having no cut-off in terms of minimum duration of treatment, the study may include trials that were underpowered, which in turn may dilute any effect.

- Since some trials are still ongoing and some trials have been discontinued without results being published, relevant data will be missed despite an extensive search.

and maintain good control of symptoms, prevent loss of lung function and minimise future risk of exacerbations and adverse effects of treatment. ${ }^{23}$ Inhaled corticosteroids (ICS) are the mainstay of pharmacotherapy for good asthma control in most patients. ${ }^{4}$ However, in approximately $10 \%$ of patients with asthma, even maximal ICS therapy does not ensure adequate control. ${ }^{5}$ A large-scale global insight study demonstrated a higher use of quick-relief medication (short-acting bronchodilators) compared with preventative medication across all asthma severities, ${ }^{6}$ indicating an unmet medical need and poor asthma control in general patient population. 
As asthma is primarily a 'type 2' inflammatory disorder, new anti-inflammatory therapeutic strategies targeting this underlying pathophysiology such as the monoclonal antibodies directed against interleukin (IL)-5, IL-4 and IL-13 signalling have been successfully developed with some of them found to be highly efficacious and safe. ${ }^{78}$

A number of in vitro studies, as well as animal and human investigations, have strongly implicated the chemoattractant receptor-homologous molecule expressed on Th2 cells (CRTH2) receptor in the pathophysiology of asthma. ${ }^{9-11}$ CRTH2 is a G-protein-coupled receptor selectively expressed by type $2 \mathrm{~T}$ lymphocytes (Th2 and Tc2), eosinophils, basophils and type 2 innate lymphoid cells (ILC2s) ${ }^{12-15}$ Mediated by its ligand prostaglandin $\mathrm{D}_{2}$, CRTH2 signalling highly promotes the recruitment and activation of eosinophils and basophils and stimulates Th2 cells and ILC2 cells to release the type 2 cytokines including IL-4, IL-5 and IL-13, leading to the development, amplification and persistence of type 2 inflammation. ${ }^{16-18}$ The CRTH2 receptor is therefore a promising new target in asthma, leading to the development of CRTH2 antagonists. ${ }^{19-21}$ In vivo and in vitro observations have highlighted the appealing therapeutic potential of CRTH2 antagonists in suppressing airway inflammation in asthma ${ }^{22-25}$ and provided a sound biological rational for its development in clinic. Until very recently, more than 20 of potent, orally bioavailable, small-molecule, competitive CRTH2 antagonists have been taken into clinical trials. ${ }^{26}$ However, the clinical results have not been consistent. Some CRTH2 antagonists are developed into the late phase of clinical trials with good safety profile and promising effect in improving lung function and patient-reported outcomes and reducing exacerbations. ${ }^{1127} 28$ While some others had been discontinued in clinical development due to low effect, ${ }^{29}$ undesirable tolerability or pharmacokinetics profiles. ${ }^{26}{ }^{30-32}$ As asthma is clinically heterogeneous with variable responses to treatment, a large interpatient variability in response to CRTH2 antagonists is also seen. It has been reported that CRTH2 antagonists contribute better improvement in lung function and asthma control in patients with greater severity of airflow limitation (forced expiratory volume in $1 \mathrm{~s}(\mathrm{FEV} 1)<70 \%)$ and in patients with atopic status. ${ }^{33} 34$ In summary, the available studies pose a lot uncertainties regarding the efficacy and safety of CRTH2 antagonists in the treatment of asthma.

\section{Aim}

We will undertake a systematic review and meta-analysis to summarise the evidence on the efficacy and safety of CRTH2 antagonists in patients with asthma from randomised controlled trials (RCTs). In addition, we hope to identify which asthma subgroup is most likely to show a clinically meaningful treatment response to this class of drug.

\section{METHODS}

This protocol has followed the Preferred Reporting Items for Systematic Review and Meta-analysis Protocols (PRISMA-P) 2015 checklist. ${ }^{35}$ Please refer to the PRISMA-P checklist in online supplementary file 1.

\section{Eligibility criteria}

Study designs

Only RCTs at least single blind with either parallel or cross-over design will be included. Observational studies (cross-sectional, case-control and cohort), case reports, case series, review articles, experimental studies with animals or in vitro, proceedings, editorials/commentaries, letters as well as policy reports comprising insufficient data to estimate the outcomes will be excluded.

\section{Participants}

Studies that recruited participants with a physician-diagnosed asthma (as per Global Initiative for Asthma (GINA) guidelines) are eligible for inclusion. ${ }^{36}$ Studies that included patients who have a significant non-asthma comorbidities will be excluded.

\section{Interventions}

A comparison between a single or split dose of oral CRTH2 antagonist and placebo will be included, consecutive use of conventional therapy (eg, ICS or long-term inhaled bronchodilator) or first aid medicine (eg, shortterm inhaled bronchodilator) as required are permitted in each group. Use of oral corticosteroid is not allowed within at least 6 weeks before and during the study period. ${ }^{29}$ Studies using allergen challenge models will be excluded.

\section{Outcomes}

Primary outcomes of interest: (1) percentage change from baseline in prebronchodilator FEV1. FEV1 is calculated as the volume of air forcibly exhaled in $1 \mathrm{~s}$ as measured by a spirometer. ${ }^{37}$ Baseline is defined as the last available FEV1 measurement taken prior to the first dose of randomised study drug; (2) asthma control, which is assessed with Asthma Control Questionnaire (ACQ) ${ }^{38} 39$ or Asthma Control Test. ${ }^{40}$ Data of the shorten ACQs, such as the fiveitem and six-item versions (ACQ-5 and ACQ-6) without assessing the last one or two items are also collected in view of their high agreement with the original one ${ }^{41}$; (3) asthma exacerbations, defined as a deterioration in symptoms requiring other medical intervention and/or hospitalisation and/or a decline in morning peak expiratory flow of $>30 \%$ from baseline on two consecutive days.

Secondary outcomes of interest: (1) adverse events (AEs). Dropouts due to side effect will also be compared between groups; (2) quality of life, which is recorded using the Standardised Asthma Quality-of-Life Questionnaire (AQLQ(S)) with four domains (symptoms, activity limitation, emotional function and environmental stimuli) comprising 32 items are evaluated ${ }^{42}$; (3) peripheral blood eosinophil count; (4) fractional exhaled nitric oxide (FENO) value. 


\section{Study search}

To identify candidate studies, a literature search will be performed in PubMed/Medline, Embase, Web of Science, Cochrane Database of Systematic Reviews, Global Index Medicus, Cochrane Central Register of Controlled Studys and Scopus for abstracts and full articles from inception to 30 December 2017. In addition, we will manually search other resources such as abstracts from major international respiratory conferences (European Respiratory Society and American Thoracic Society, 2001-2017), key journals and the reference list of all the included articles. ClinicalStudys.gov (http://www. clinicalstudys.gov), metaRegister of Controlled Studys (http://www.controlled-studys.com), ISRCTN registry (https://www.isrctn.com) and European Clinical Trials Database (https://eudract.ema.europa.eu/index.html) will also be searched for ongoing studies. For ongoing reports or studies published in abstract form, the primary author will be contacted by email to request the required data as necessary.

\section{Search strategy}

A search strategy based on the combination of relevant terms will be applied, with vocabulary and syntax adjusted across databases. Our search strategy includes the terms 'asthma' AND 'Prostaglandin D2 receptor 2' OR 'PTGDR2' OR 'chemoattractant Receptor-Homologous Molecule Expressed on Th2 Cells' OR 'CRTH2' OR 'D-type prostanoid receptor' OR 'DP2' AND 'antagonist' OR 'inhibitor' OR 'blocker'. Specific names of CRTH2 antagonists including 'OC000459' or 'OC459', 'QAW039' or 'fevipiprant', 'AZD1981', 'BI 671800', 'AMG 853', 'ACT-129968', 'ASP5642', 'ARRY-502', 'AZD6430', 'ADC3680', 'MK-1029', 'MK-7246', 'ACT-129968' or 'KYTH105 ' or 'setipiprant', 'BI 1021958' and so on, will be searched in combined with 'asthma" for relevant studies. Full search strategy used in Pubmed/Medline is provided in online supplementary file 2 .

\section{Study selection}

The selection phase will be illustrated using a PRISMA flow diagram, ${ }^{4}$ with reasons specified for excluding certain articles being recorded. Studies identified with electronic and manual searches will be saved and deduplicated in EndNote. Literature search results will be uploaded to Covidence, an online software used to minimise bias and errors in study screening and data extraction. The inclusion and exclusion criteria used for screening will be pilot tested on a subset of search results. The study selection will be performed in three steps. First, two independent reviewers (WL and JM) will screen the titles and abstracts of all records identified by the search strategy in line with the inclusion/exclusion criteria to identify potentially eligible articles. Second, full texts of these articles will be obtained and assessed by the same two independent reviewers. Third, the screening results will be cross-checked. Any discrepancies relating to study selection will be resolved by consensus and/or by a third party $(\mathrm{HJ})$. Agreement in title/abstract and full-text inclusion will be reported using Cohen's K statistic. Members of the review team will be trained in software use before starting the review.

\section{Data extraction}

A standard data extraction form will be designed by the principal investigator (WL) and be pilot tested on a small number of articles in Covidence. The form will be refined according to the results of the pilot test to ensure the reliability and completeness of extracted data and to facilitate the collection process. Data extraction will be performed by two independent authors (WL and JM), with a third author acting as an arbiter when consensus is not reached $(\mathrm{HJ})$. A training session will be provided for all researchers involved in data coding if necessary. The information attempt to be extracted from all included studies, as/if available, is presented below:

- Study: first author, year of publication, journal, publication status, country/countries, start year, study stage, registration identification, sponsor/developer, funding, centres.

- Method: study design, duration of follow-up.

- Participants: sample size, age (range, mean or mean $\pm \mathrm{SD}$ ), gender distribution, method to establish the diagnosis of asthma, asthma severity, medication status, baseline sputum eosinophil percentage or blood eosinophil count, atopic/allergic status (result of skin prick test or radioallergosorbent test, history of food/drug allergy or other allergic diseases), dropouts.

- Interventions: intervention name, dose, frequency, treatment duration, wash-out period.

- Outcomes: percentage change from baseline in $\mathrm{FEV}_{1}$, ACQ score, exacerbation frequency, AEs frequency, AQLQ(S) score, blood eosinophil count, FENO value.

- Other information: conflicts of interest, funding.

As studies may sometimes be reported in several publications, we will extract data from the largest sample when possible. Corresponding authors of the original studies will be contacted to clarify article eligibility if necessary.

\section{Management of missing data}

If data are not directly reported, primary authors will be contacted. Otherwise, analysis will be conducted on the final available data, and the potential impact of the missing data will be discussed as a limitation. The proportion of missing data will be reported along with reasons, where indicated.

\section{Quality assessment}

The risk of bias of included RCTs will be assessed using the Cochrane Collaboration risk of bias assessment tool and reported on a per-study basis. ${ }^{44}$ Two independent review authors (WL and JM) will perform the study quality assessment separately. The quality assessment tool includes seven specific domains as follows: random sequence generation (selection bias), allocation 
concealment (selection bias), blinding of participants and personnel (performance bias), blinding of outcome assessment (detection bias), incomplete outcome data (attrition bias), selective reporting (reporting bias) and other sources of bias (other bias). Each domain will be categorised as 'low risk of bias', 'high risk of bias' or 'unclear risk of bias'. Figures of risk of bias will be generated using Review Manager (RevMan) V.5.3.5 (Cochrane Informatics and Knowledge Management Department). ${ }^{45}$

\section{Data synthesis and analysis}

For all primary and secondary outcomes in our study, relative risks and weighted mean differences with the corresponding $95 \%$ CIs will be calculated for dichotomous (binary) data and continuous data, respectively. Statistical analysis of outcomes will be based on 'intention-to-treat' principle.

In line with standard methodology, ${ }^{44}$ the statistical heterogeneity between individual studies will be assessed by the $\chi^{2}$ test for the Cochran's $Q$ (statistically significant at $\mathrm{p}<0.10)$ and quantified by the $\mathrm{I}^{2}$ statistics $(25 \%$, $50 \%$ and $75 \%$ is categorised as low, moderate and high heterogeneity, respectively) ${ }^{46}$ Assuming the existence of variations across individual RCTs in patient population, follow-up duration, type and dosage of CRTH2 antagonist and the measurement or report methods on outcomes, the random-effects model (DerSimonian-Laird method) will be used. A narrative, qualitative synthesis will be done instead of a meta-analysis if between-study heterogeneity is high or there is no enough available data for any comparison.

Where significant heterogeneity is present, subgroup analyses for the primary outcomes will be conducted. The following covariates expected to account for clinical or methodological heterogeneity will be evaluated, as/if available, in subgroup analyses:

- Type of each specific CRTH2 antagonist.

- Pharmacological characteristics of CRTH2 antagonists: the medications are classified into selective CRTH2 antagonist alone or non-selective CRTH2 antagonist including CRTH2/D-type prostanoid dual antagonist and CRTH2/thromboxane prostanoid dual antagonist.

- Inflammatory phenotype (eosinophilic or non-eosinophilic): patients with a baseline proportion of sputum eosinophils $\geq 2 \%$ of all sputum inflammatory cells ${ }^{47}$ or a baseline blood eosinophil count $\geq 150$ cells $/ \mu \mathrm{L}$ and/ or a historical blood eosinophil count $\geq 300$ cells $/ \mu \mathrm{L}$ are defined as eosinophilic asthmatics, ${ }^{48}$ despite corticosteroid therapy.

- Disease severity according to GINA guideline. ${ }^{36}$

- Atopic/allergic status (allergic or non-allergic): the atopic/allergic status is defined according to the history of food/drug allergy and/or other allergic disease (such as eczema, allergic rhinitis or atopic dermatitis) and/or the result of allergic examination (skin prick testing, radioallergosorbent test, etc).
We acknowledge the potential for outliers among the larger collection of studies with respect to the effect estimates. Therefore, a leave-one-out sensitivity analysis will be conducted to identify the impact of each study on the summary estimates, where substantial variations will prompt the investigation of the outlier, with a particular focus on possible factor that explain the variation. RevMan V.5.3.5 will be used for all analyses. Studies will be presented as a summary table and forest $\operatorname{plot}(\mathrm{s})$.

\section{Publication bias assessment}

Publication bias will be investigated using the funnel plot test if the number of articles is $\geq 10$. If asymmetry is showed in the funnel plot, Egger's regression test and Duval and Tweedie trim-and-fill method will be performed. ${ }^{49} 50$ Publication bias will be assumed at $p$ value $<0.1$. In addition, contour-enhanced meta-analysis funnel plots will be conducted to distinguish publication bias from other causes of asymmetry. ${ }^{51}$ Publication bias assessment will be generated by STATA (12.0).

\section{Evaluating the quality of evidence}

The quality of evidence for outcomes will be assessed using the approach specified by the 'Grading of Recommendations Assessment, Development and Evaluation' (GRADE). ${ }^{44}{ }^{52}$ The GRADE judgement takes several factors into account including the risk of bias, imprecision, inconsistency, size of the effect, effect of residual confounding, presence of a dose-response relationship and publication bias. The collective effects of these factors are combined to arrive at a conclusion regarding the quality of the overall body of evidence rather than individual studies. Based on the GRADE assessment, the quality of the body of evidence will be designated into one of the following categories:

- High ( $\geq 4$ points): we are confident that the true effect lies close to that of the estimate of the effect.

- Moderate (three points): the true effect is likely to be close to the estimate of the effect; however, there is a chance that it is considerably different.

- Low (two points): confidence in the estimate of the true effect is limited; the true effect may be substantially different from the estimate.

- Very low $(\leq 1$ point): we have very little confidence in the estimated effect; it is likely to be significantly different from the true effect.

\section{Ethics and dissemination}

Ethical approval is not required for this systematic review since the data will be anonymised. Results of this systematic review will be disseminated via publication in a peer-reviewed journal and conference presentations.

\section{Patient and public involvement}

No patients or public were involved in this study. 


\section{DISCUSSION}

Asthma continues to be a significant health concern. In the current era of undertreatment, the development of effective drugs for patients with asthma remains an important focus for respiratory researchers and clinicians. As a new emerging treatment, orally bioavailable CRTH2 antagonists have been in clinical trials to assess their safety and efficacy. However, the evidence from available studies has been conflicting. A comprehensive systematic review may play an important role in increasing scientific certainty, informing clinical decisions and subsequent studies. The current protocol is designed for the first systematic review and meta-analysis to synthesise evidence on the efficacy and safety of CRTH2 antagonists in patients with asthma.

Studies in asthma have shown a strong correlation between increasing levels of CRTH2 expression and significant blood and sputum eosinophilia. ${ }^{9}$ Eosinophilic inflammation has been associated with more severe asthma and atopic conditions in general asthma populations. ${ }^{53} 54$ In the current review, we extract details about inflammatory phenotype, disease severity and atopic status to try to evaluate the influence of these variables on the efficacy of CRTH2 antagonist by stratified analyses. Results of this review will define patients who are most likely to achieve clinically significant benefit with CRTH2 antagonist treatment, which may finally enhance the care provided to patients with asthma.

Contributors WL and JM were first coauthors and they contributed equally to this work. WL and BM conceived and designed the protocol. WL and JM piloted the data extraction forms and drafted the manuscript. All authors critically revised the manuscript for methodological content and approved the final version of this manuscript. $\mathrm{HJ}$ is the guarantor of the review.

Funding This research received no specific grant from any funding agency in the public, commercial or not-for-profit sectors.

Competing interests None declared.

Patient consent Not required.

Provenance and peer review Not commissioned; externally peer reviewed.

Open Access This is an Open Access article distributed in accordance with the Creative Commons Attribution Non Commercial (CC BY-NC 4.0) license, which permits others to distribute, remix, adapt, build upon this work non-commercially, and license their derivative works on different terms, provided the original work is properly cited and the use is non-commercial. See: http://creativecommons.org/ licenses/by-nc/4.0/

(c) Article author(s) (or their employer(s) unless otherwise stated in the text of the article) 2018. All rights reserved. No commercial use is permitted unless otherwise expressly granted.

\section{REFERENCES}

1. Becker AB, Abrams EM. Asthma guidelines: the global initiative for asthma in relation to national guidelines. Curr Opin Allergy Clin Immunol 2017;17:99-103.

2. GINA. GIfA. Global strategy for asthma management and prevention. 2014 http://wwwginasthmaorg

3. British Thoracic SocietyScottish Intercollegiate Guidelines Network. British guideline on the management of asthma. Thorax 2014;69(Suppl 1):1-192.

4. Murphy KR, Bender BG. Treatment of moderate to severe asthma: patient perspectives on combination inhaler therapy and implications for adherence. J Asthma Allergy 2009;2:63-72.
5. Rabe KF, Adachi M, Lai CK, et al. Worldwide severity and control of asthma in children and adults: the global asthma insights and reality surveys. J Allergy Clin Immunol 2004;114:40-7.

6. American Thoracic Society. Proceedings of the ATS workshop on refractory asthma: current understanding, recommendations, and unanswered questions. Am J Respir Crit Care Med 2000;162:2341-51

7. Farne $\mathrm{H}$, Jackson DJ, Johnston SL. Are emerging PGD2 antagonists a promising therapy class for treating asthma? Expert Opin Emerg Drugs 2016;21:359-64.

8. Walsh GM. Biologics targeting IL-5, IL-4 or IL-13 for the treatment of asthma: an update. Expert Rev Clin Immunol 2017;13:143-9.

9. Fajt ML, Gelhaus SL, Freeman B, et al. Prostaglandin D $\square$ pathway upregulation: relation to asthma severity, control, and $\mathrm{TH} 2$ inflammation. J Allergy Clin Immunol 2013;131:1504-12.

10. Pettipher R, Hunter MG, Perkins CM, et al. Heightened response of eosinophilic asthmatic patients to the CRTH2 antagonist OC000459. Allergy 2014;69:1223-32.

11. Barnes N, Pavord I, Chuchalin A, et al. A randomized, double-blind, placebo-controlled study of the CRTH2 antagonist OC000459 in moderate persistent asthma. Clin Exp Allergy 2012;42:38-48.

12. Hirai H, Tanaka K, Yoshie O, et al. Prostaglandin D2 selectively induces chemotaxis in T helper type 2 cells, eosinophils, and basophils via seven-transmembrane receptor CRTH2. J Exp Med 2001;193:255-61.

13. Nagata K, Tanaka K, Ogawa K, et al. Selective expression of a novel surface molecule by human Th2 cells in vivo. $\mathrm{J}$ Immunol 1999;162:1278-86.

14. O'Byrne PM, Naji N, Gauvreau GM. Severe asthma: future treatments. Clin Exp Allergy 2012;42:706-11.

15. Xue L, Salimi M, Panse I, et al. Prostaglandin D2 activates group 2 innate lymphoid cells through chemoattractant receptor-homologous molecule expressed on TH2 cells. J Allergy Clin Immunol 2014;133:1184-94

16. Miadonna A, Tedeschi A, Brasca C, et al. Mediator release after endobronchial antigen challenge in patients with respiratory allergy. $J$ Allergy Clin Immunol 1990;85:906-13.

17. Turner NC, Fuller RW, Jackson DM. Eicosanoid release in allergeninduced bronchoconstriction in dogs. Its relationship to airways hyperreactivity and pulmonary inflammation. J Lipid Mediat Cell Signal 1995;11:93-102.

18. Monneret G, Gravel S, Diamond M, et al. Prostaglandin D2 is a potent chemoattractant for human eosinophils that acts via a novel DP receptor. Blood 2001;98:1942-8.

19. Schuligoi R, Sturm E, Luschnig P, et al. CRTH2 and D-type prostanoid receptor antagonists as novel therapeutic agents for inflammatory diseases. Pharmacology 2010;85:372-82.

20. Norman P. DP(2) receptor antagonists in development. Expert Opin Investig Drugs 2010;19:947-61.

21. Pettipher R, Hansel TT, Armer R. Antagonism of the prostaglandin D2 receptors DP1 and $\mathrm{CRTH} 2$ as an approach to treat allergic diseases. Nat Rev Drug Discov 2007;6:313-25.

22. Lukacs NW, Berlin AA, Franz-Bacon K, et al. CRTH2 antagonism significantly ameliorates airway hyperreactivity and downregulates inflammation-induced genes in a mouse model of airway inflammation. Am J Physiol Lung Cell Mol Physiol 2008;295:L767-79.

23. Uller L, Mathiesen JM, Alenmyr L, et al. Antagonism of the prostaglandin D2 receptor CRTH2 attenuates asthma pathology in mouse eosinophilic airway inflammation. Respir Res 2007;8:16.

24. Sidharta PN, Diamant Z, Dingemanse J. Single- and multipledose tolerability and pharmacokinetics of the CRTH2 antagonist setipiprant in healthy male subjects. Fundam Clin Pharmacol 2014;28:690-9.

25. Horak F, Zieglmayer P, Zieglmayer R, et al. The CRTH2 antagonist OC000459 reduces nasal and ocular symptoms in allergic subjects exposed to grass pollen, a randomised, placebo-controlled, doubleblind trial. Allergy 2012;67:1572-9.

26. Norman P. Update on the status of DP2 receptor antagonists: from proof of concept through clinical failures to promising new drugs. Expert Opin Investig Drugs 2014;23:55-6.

27. Bateman ED, Guerreros AG, Brockhaus F, et al. Fevipiprant, an ora prostaglandin $\mathrm{DP}_{2}$ receptor $\left(\mathrm{CRTh}_{2}\right)$ antagonist, in allergic asthma uncontrolled on low-dose inhaled corticosteroids. Eur Respir J 2017;50:1700670.

28. Hall IP, Fowler AV, Gupta A, et al. Efficacy of BI 671800, an oral CRTH2 antagonist, in poorly controlled asthma as sole controller and in the presence of inhaled corticosteroid treatment. Pulm Pharmacol Ther 2015;32:37-44.

29. Busse WW, Wenzel SE, Meltzer EO, et al. Safety and efficacy of the prostaglandin D2 receptor antagonist AMG 853 in asthmatic patients. J Allergy Clin Immunol 2013;131:339-45. 
30. Jeremy IL, Stefan L. Anti-inflammatory drug discovery. Royal Society of Chemistry 2012:125-7.

31. Wang YH, Trucksis M, McElwee JJ, et al. UGT2B17 genetic polymorphisms dramatically affect the pharmacokinetics of MK-7246 in healthy subjects in a first-in-human study. Clin Pharmacol Ther 2012;92:96-102.

32. Bain G, King CD, Brittain J, et al. Pharmacodynamics, pharmacokinetics, and safety of AM211: a novel and potent antagonist of the prostaglandin D2 receptor type 2. J Clin Pharmacol 2012;52:1482-93.

33. Erpenbeck VJ, Popov TA, Miller D, et al. The oral CRTh2 antagonist QAW039 (fevipiprant): a phase II study in uncontrolled allergic asthma. Pulm Pharmacol Ther 2016;39:54-63.

34. Kuna P, Bjermer L, Tornling G. Two phase II randomized trials on the CRTh2 antagonist AZD1981 in adults with asthma. Drug Des Devel Ther 2016;10:2759-70.

35. Moher D, Shamseer L, Clarke M, et al. Preferred reporting items for systematic review and meta-analysis protocols (PRISMA-P) 2015 statement. Syst Rev 2015;4:1.

36. Global Initiative for Asthma. Global strategy for asthma management and prevention. 2017 wwwginasthmaorg

37. Miller MR, Hankinson J, Brusasco V, et al. Standardisation of spirometry. Eur Respir J 2005;26:319-38.

38. Juniper EF, O'Byrne PM, Guyatt GH, et al. Development and validation of a questionnaire to measure asthma control. Eur Respir $J$ 1999:14:902-7.

39. Barnes PJ, Casale TB, Dahl R, et al. The Asthma Control Questionnaire as a clinical trial endpoint: past experience and recommendations for future use. Allergy 2014;69:1119-40.

40. Nathan RA, Sorkness CA, Kosinski M, et al. Development of the asthma control test: a survey for assessing asthma control. J Allergy Clin Immunol 2004;113:59-65.

41. Juniper EF, Svensson K, Mörk AC, et al. Measurement properties and interpretation of three shortened versions of the asthma control questionnaire. Respir Med 2005;99:553-8.
42. Juniper EF, Buist AS, Cox FM, et al. Validation of a standardized version of the Asthma Quality of Life Questionnaire. Chest 1999;115:1265-70.

43. Moher D, Liberati A, Tetzlaff J, et al. Preferred reporting items for systematic reviews and meta-analyses: the PRISMA statement. PLoS Med 2009;6:e1000097.

44. Higgins J, Green S. Cochrane handbook for systematic reviews of interventions version 5.1.0: The Cochrane collaboration, 2011.

45. Collaboration NCCTC. Review Manager (RevMan) [computer program] Version 53. Copenhagen: The Nordic Cochrane Centre, The Cochrane Collaboration, 2014.

46. Higgins JP, Thompson SG, Deeks JJ, et al. Measuring inconsistency in meta-analyses. BMJ 2003;327:557-60.

47. Wenzel SE. Asthma phenotypes: the evolution from clinical to molecular approaches. Nat Med 2012;18:716-25.

48. Yancey SW, Keene ON, Albers FC, et al. Biomarkers for severe eosinophilic asthma. J Allergy Clin Immunol 2017;140:1509-18.

49. Egger M, Davey Smith G, Schneider M, et al. Bias in meta-analysis detected by a simple, graphical test. BMJ 1997;315:629-34.

50. Duval S, Tweedie R. Trim and fill: a simple funnel-plot-based method of testing and adjusting for publication bias in meta-analysis. Biometrics 2000;56:455-63.

51. Peters JL, Sutton AJ, Jones DR, et al. Contour-enhanced metaanalysis funnel plots help distinguish publication bias from other causes of asymmetry. J Clin Epidemiol 2008;61:991-6.

52. Guyatt G, Oxman AD, Akl EA, et al. GRADE guidelines: 1. Introduction-GRADE evidence profiles and summary of findings tables. J Clin Epidemiol 2011;64:383-94.

53. Woodruff PG, Khashayar R, Lazarus SC, et al. Relationship between airway inflammation, hyperresponsiveness, and obstruction in asthma. J Allergy Clin Immunol 2001;108:753-8.

54. Tran TN, Zeiger RS, Peters SP, et al. Overlap of atopic, eosinophilic, and TH2-high asthma phenotypes in a general population with current asthma. Ann Allergy Asthma Immunol 2016;116:37-42 